\title{
Galerkin Method for the numerical solution of the RLW equation by using exponential B-splines
}

\author{
M. Z. Görgülü, İ. Dağ and D. Irk \\ Department of Mathematics-Computer Science, Eskisehir Osmangazi \\ University, 26480, Eskisehir, Turkey.
}

\begin{abstract}
In this paper, the exponential B-spline functions are used for the numerical solution of the RLW equation. Three numerical examples related to propagation of single solitary wave, interaction of two solitary waves and wave generation are employed to illustrate the accuracy and the efficiency of the method. Obtained results are compared with some early studies.
\end{abstract}

Keywords: Exponential B-spline; Galerkin Method; RLW equation.

\section{Introduction}

Many studies exist for the numerical solutions of the differential equations using splines. Splines are piecewise functions which have certain continuity at the joint points given to set up the splines. The spline related numerical techniques mainly offer the economical computer code and easy computational calculations. Thus they are preferable in forming the numerical methods. Until now, polynomial splines have been extensively developed and used for approximation of curve and surfaces and finding solutions of the differential equations. The polynomial spline based algorithms have been found to be quite advantageous for finding solutions of the differential equations. Because it has been demonstrated that they yield the lower cost and simplicity to write the program code. Base of splines known as the B-splines is also widely used to build up the trial functions for numerical methods. The exponential spline is proposed to be more general form of these splines. In the approximation theory, the exponential B-splines are shown to model the data which have sudden growth and decay whereas polynomials are not appropriate due to having osculatory behavior. Since some differential equations 
have steep solutions, the use of the exponential B-splines in the numerical methods may exhibit good solutions for differential equations. McCartin [26] has introduced the exponential B-spline as a basis for the space of exponential splines. The exponential B-spline properties accord with those of polynomial B-splines such as smootness, compact support, positivity, recursion for derivatives. Thus the exponential B-splines can be used as the trial function for the variational methods such as Galerkin and collocation methods.

The exponential B-spline based methods have been started to solve some differential equations: Numerical solution of the singular perturbation problem is solved with a variant of exponential B-spline collocation method in the work [7], the cardinal exponential B-splines is used for solving the singularly perturbed problems [32], the exponential B-spline collocation method is built up for finding the numerical solutions of the self-adjoint singularly perturbed boundary value problems in the work [31, the numerical solutions of the Convection-Diffusion equation is obtained by using the exponential B-spline collocation method [33].

The collocation methods based on the exponential B-spline functions have been constructed to solve the differential equations. In this study, the exponential B-spline function are used to set up the trial functions which are placed in place of the unknown variable of the differential equations for the Galerkin finite element method. Thus nonlinear RLW equation will be solved with the proposed method numerically. The RLW equation describes a large number of important physical phenomena, such as shallow waters and plasma waves. Therefore it plays a major role in the study of nonlinear dispersive waves. Because of having limited analytical solutions, numerical analysis of the RLW equation has an importance in its study.

Various techniques have been developed to obtain the numerical solution of this nonlinear partial differential equation, some of which are finite difference methods [19, 3, 8, 11, 15, finite element methods [2, 20, 17, 22, 24, 25, 12, 16, 13, 14, 23] and spectral methods [6, 21, 15.

The paper is outlined as follows. In section 2, exponential B-splines and their some basic relations are introduced. In section 3, the application of the numerical method is given. The efficiency and the accuracy of the present method are investigated by using three numerical experiments related to propagation of single solitary wave, interaction of two solitary waves and wave generation. Finally some remarks are concluded in the last section. 


\section{Exponential B-splines and Finite Element Solu- tion}

In this study, we will consider the regularized long wave (RLW) equation

$$
u_{t}+u_{x}+\varepsilon u u_{x}-\mu u_{x x t}=0
$$

where $x$ is space coordinate, $t$ is time, $u$ is the wave amplitude and $\varepsilon$ and $\mu$ are positive parameters. Boundary and initial conditions of the Eq.(1) are

$$
\begin{aligned}
& u(a, t)=\beta_{1}, u(b, t)=\beta_{2} \\
& u_{x}(a, t)=0, u_{x}(b, t)=0, t \in(0, T], \\
& u(x, 0)=f(x), x \in[a, b] .
\end{aligned}
$$

This equation (11) was first introduced by Peregrine [19] for modelling the propagation of unidirectional weakly nonlinear and weakly dispersive water waves. Since the RLW equation obviates the certain problematical aspects of $\mathrm{KdV}$ equation and it generally has more expedient mathematical properties, Benjamin et al. [1] proposed the use of RLW equation as a preferable model to the $\mathrm{KdV}$ equation.

Let us consider a uniform mesh $\Gamma$ with the knots $x_{i}$ on $[a, b]$ such that

$$
\Gamma: a=x_{0}<x_{1}<x_{2}<\cdots<x_{N-1}<x_{N}=b
$$

where $h=\frac{b-a}{N}$ and $x_{i}=x_{0}+i h$.

Let $B_{i}(x)$ be the B-splines at the points of $\Gamma$ together with knots $x_{i}$, $i=-3,-2,-1, N+1, N+2, N+3$ outside the interval $[a, b]$ and having a finite support on the four consecutive intervals $\left[x_{i}+k h, x_{i}+(k+1) h\right]_{k=-3}^{0}$, $i=0, \ldots, N+2$. The $B_{i}(x)$ can be defined as

$B_{i}(x)= \begin{cases}b_{2}\left[\left(x_{i-2}-x\right)-\frac{1}{p}\left(\sinh \left(p\left(x_{i-2}-x\right)\right)\right)\right] & \text { if } x \in\left[x_{i-2}, x_{i-1}\right] \\ a_{1}+b_{1}\left(x_{i}-x\right)+c_{1} e^{p\left(x_{i}-x\right)}+d_{1} e^{-p\left(x_{i}-x\right)} & \text { if } x \in\left[x_{i-1}, x_{i}\right] \\ a_{1}+b_{1}\left(x-x_{i}\right)+c_{1} e^{p\left(x-x_{i}\right)}+d_{1} e^{-p\left(x-x_{i}\right)} & \text { if } x \in\left[x_{i}, x_{i+1}\right] \\ b_{2}\left[\left(x-x_{i+2}\right)-\frac{1}{p}\left(\sinh \left(p\left(x-x_{i+2}\right)\right)\right)\right] & \text { if } x \in\left[x_{i+1}, x_{i+2}\right] \\ 0 & \text { otherwise. }\end{cases}$

where 


$$
\begin{aligned}
& p=\max _{0 \leq i \leq N} p_{i}, s=\sinh (p h), c=\cosh (p h) \\
& b_{2}=\frac{p}{2(p h c-s)}, a_{1}=\frac{p h c}{p h c-s}, b_{1}=\frac{p}{2}\left[\frac{c(c-1)+s^{2}}{(p h c-s)(1-c)}\right], \\
& c_{1}=\frac{1}{4}\left[\frac{e^{-p h}(1-c)+s\left(e^{-p h}-1\right)}{(p h c-s)(1-c)}\right], d_{1}=\frac{1}{4}\left[\frac{e^{p h}(c-1)+s\left(e^{p h}-1\right)}{(p h c-s)(1-c)}\right]
\end{aligned}
$$

Each basis function $B_{i}(x)$ is twice continuously differentiable. The values of $B_{i}(x), B_{i}^{\prime}(x)$ and $B_{i}^{\prime \prime}(x)$ at the knots $x_{i}$ 's are obtained from the Table 1 .

Table 1: Exponential B-spline values

\begin{tabular}{|cccccc|}
\hline & $x_{i-2}$ & $x_{i-1}$ & $x_{i}$ & $x_{i+1}$ & $x_{i+2}$ \\
\hline$B_{i}(x)$ & 0 & $\frac{s-p h}{2(p h c-s)}$ & 1 & $\frac{s-p h}{2(p h c-s)}$ & 0 \\
$B_{i}^{\prime}(x)$ & 0 & $\frac{p(1-c)}{2(p h c-s)}$ & 0 & $\frac{p(c-1)}{2(p h c-s)}$ & 0 \\
$B_{i}^{\prime \prime}(x)$ & 0 & $\frac{p^{2} s}{2(p h c-s)}$ & $\frac{-p^{2} s}{p h c-s}$ & $\frac{p^{2} s}{2(p h c-s)}$ & 0 \\
\hline
\end{tabular}

The $B_{i}(x), i=-1, \ldots, N+1$ form a basis for functions defined on the interval $[a, b]$. We seek an approximation $U_{N}(x, t)$ to the analytical solution $u(x, t)$ in terms for the exponential B-splines

$$
u(x, t) \approx U_{N}(x, t)=\sum_{i=-1}^{N+1} B_{i}(x) \delta_{i}(t)
$$

where $\delta_{i}(t)$ are time dependent unknown to be determined from the boundary conditions and Galerkin approach to the equation (1). The approximate solution and their derivatives at the knots can be found from the Eq. (3-4) as

$$
\begin{aligned}
& U_{i}=U_{N}\left(x_{i}, t\right)=\alpha_{1} \delta_{i-1}+\delta_{i}+\alpha_{1} \delta_{i+1}, \\
& U_{i}^{\prime}=U_{N}^{\prime}\left(x_{i}, t\right)=\alpha_{2} \delta_{i-1}-\alpha_{2} \delta_{i+1} \\
& U_{i}^{\prime \prime}=U_{N}^{\prime \prime}\left(x_{i}, t\right)=\alpha_{3} \delta_{i-1}-2 \alpha_{3} \delta_{i}+\alpha_{3} \delta_{i+1}
\end{aligned}
$$

where $\alpha_{1}=\frac{s-p h}{2(p h c-s)}, \alpha_{2}=\frac{p(1-c)}{2(p h c-s)}, \alpha_{3}=\frac{p^{2} s}{2(p h c-s)}$.

Applying the Galerkin method to the RLW equation with the exponential B-splines as weight function over the element $[a, b]$ gives

$$
\int_{a}^{b} B_{i}(x)\left(u_{t}+u_{x}+\varepsilon u u_{x}-\mu u_{x x t}\right) d x=0 .
$$

The approximate solution $U_{N}$ over the element $\left[x_{m}, x_{m+1}\right]$ can be written as 
$U_{N}^{e}=B_{m-1}(x) \delta_{m-1}(t)+B_{m}(x) \delta_{m}(t)+B_{m+1}(x) \delta_{m+1}(t)+B_{m+2}(x) \delta_{m+2}(t)$

where quantities $\delta_{j}(t), j=m-1, \ldots, m+2$ are element parameters and $B_{j}(x), j=m-1, \ldots, m+2$ are known as the element shape functions.

The contribution of the integral equation (6) over the sample interval $\left[x_{m}, x_{m+1}\right]$ is given by

$$
\int_{x_{m}}^{x_{m+1}} B_{j}(x)\left(u_{t}+u_{x}+\varepsilon u u_{x}-\mu u_{x x t}\right) d x=0 .
$$

Applying the Galerkin discretization scheme by replacing approximate solution $U_{N}^{e}(7)$ and its derivatives $\left(U_{N}\right)_{t},\left(U_{N}\right)_{x},\left(U_{N}\right)_{x x t}$ into the exact solution $u$ and its derivatives $u_{t}, u_{x}, u_{x x t}$ respectively, we obtain a system of equations in the unknown parameters $\delta_{j}$

$$
\begin{aligned}
& \sum_{i=m-1}^{m+2}\left\{\left(\int_{x_{m}}^{x_{m+1}} B_{j} B_{i} d x\right) \dot{\delta}_{i}+\left(\int_{x_{m}}^{x_{m+1}} B_{j} B_{i}^{\prime} d x\right) \delta_{i}\right. \\
& \left.+\varepsilon\left(\int_{x_{m}}^{x_{m+1}} B_{j}\left(\sum_{k=m-1}^{m+2} \delta_{k} B_{k}\right) B_{i}^{\prime} d x\right) \delta_{i}-\mu\left(\int_{x_{m}}^{x_{m+1}} B_{j} B_{i}^{\prime \prime} d x\right) \dot{\delta}_{i}\right\}=0
\end{aligned}
$$

where $i, j$ and $k$ take only the values $m-1, m, m+1, m+2$ for $m=$ $0,1, \ldots, N-1$ and $\bullet$ denotes time derivative.

In the above system of differential equations, when $A^{e}, B^{e}, C^{e}(\delta)$ and $D^{e}$ are denoted by

$$
\begin{array}{ll}
A^{e}=\int_{x_{m}}^{x_{m+1}} B_{j} B_{i} d x, & B^{e}=\int_{x_{m}}^{x_{m+1}} B_{j} B_{i}^{\prime} d x \\
C^{e}(\delta)=\int_{x_{m}}^{x_{m+1}} B_{j}\left(\sum_{k=m-1}^{m+2} \delta_{k} B_{k}\right) B_{i}^{\prime} d x, & D^{e}=\int_{x_{m}}^{x_{m+1}} B_{j} B_{i}^{\prime \prime} d x
\end{array}
$$

where $A^{e}, B^{e}$ and $D^{e}$ are the element matrices of which dimensions are $4 \times 4$ and $C^{e}(\delta)$ is the element matrix with the dimension $4 \times 4 \times 4$, the matrix form of the Eq. (9) can be written as

$$
\left(\mathbf{A}^{e}-\mu \mathbf{D}^{e}\right) \dot{\delta}^{e}+\left(\mathbf{B}^{e}+\varepsilon \mathbf{C}^{e}\left(\delta^{e}\right)\right) \delta^{e}=0
$$

where $\delta^{e}=\left(\delta_{m-1}, \ldots, \delta_{m+2}\right)^{T}$

Gathering the systems (11) over all elements, we obtain global system

$$
(\mathbf{A}-\mu \mathbf{D}) \dot{\delta}+(\mathbf{B}+\varepsilon \mathbf{C}(\delta)) \delta=0
$$


where $\mathbf{A}, \mathbf{B}, \mathbf{C}(\delta), \mathbf{D}$ are derived from the corresponding element matrices $A^{e}, B^{e}, C^{e}(\delta), D^{e}$, respectively and $\delta=\left(\delta_{-1}, \ldots, \delta_{N+1}\right)^{T}$ contain all elements parameters.

The unknown parameters $\delta$ are interpolated between two time levels $n$ and $n+1$ with the Crank-Nicolson method

$$
\delta=\frac{\delta^{n+1}+\delta^{n}}{2}, \dot{\delta}=\frac{\delta^{n+1}-\delta^{n}}{\Delta t},
$$

we obtain iterative formula for the time parameters $\delta^{n}$

$$
\left[(\mathbf{A}-\mu \mathbf{D})+\frac{\Delta t}{2}\left(\mathbf{B}+\varepsilon \mathbf{C}\left(\delta^{n+1}\right)\right)\right] \delta^{n+1}=\left[(\mathbf{A}-\mu \mathbf{D})-\frac{\Delta t}{2}\left(\mathbf{B}+\varepsilon \mathbf{C}\left(\delta^{n}\right)\right)\right] \delta^{n}
$$

The set of equations consist of $(N+3)$ equations with $(N+3)$ unknown parameters. Before starting the iteration procedure, boundary conditions must be adapted into the system and initial vector $\delta^{n}$ must also be determined.

We delete first and last equations from the system (13) and eliminate the terms $\delta_{-1}^{n+1}$ and $\delta_{N+1}^{n+1}$ from the system (13) by using boundary conditions in (2), which give the following equations:

$u(a, t)=m_{1} \delta_{-1}^{n}+\delta_{0}^{n}+m_{1} \delta_{1}^{n}=\beta_{1}, u(b, t)=m_{1} \delta_{N-1}^{n}+\delta_{N}^{n}+m_{1} \delta_{N+1}^{n}=\beta_{1}$

we obtain a septa-diagonal matrix with the dimension $(N+1) \times(N+1)$. Then we can solve this matrix system through Thomas algorithm. Since the system 13 is an implicit system due to the term $\mathbf{C}\left(\delta^{n+1}\right)$, we have used the following inner iteration:

$$
\left(\delta^{*}\right)^{n+1}=\delta^{n+1}+\frac{\left(\delta^{n+1}-\delta^{n}\right)}{2} .
$$

In this iteration, before moving the calculation of the next time step approximation for time parameter, we calculate the new vectors $\left(\delta^{*}\right)^{n+1}$ using the formula (14) from previous vectors $\delta^{n+1}$ finding form the system (13) and then repeat three times at all time steps.

To start evolution of the vector of initial parameters $\delta^{0}$, it must be determined by using the initial condition and boundary conditions:

$$
\begin{aligned}
& u_{0}^{\prime}\left(x_{0}, 0\right)=\frac{p(1-c)}{2(p h c-s)} \delta_{-1}+\frac{p(c-1)}{2(p h c-s)} \delta_{1} \\
& u\left(x_{m}, 0\right)=\frac{s-p h}{2(p h c-s)} \delta_{m-1}+\delta_{m}+\frac{s-p h}{2(p h c-s)} \delta_{m+1}, m=0, \ldots, N \\
& u^{\prime}\left(x_{N}, 0\right)=\frac{p(1-c)}{2(p h c-s)} \delta_{N-1}+\frac{p(c-1)}{2(p h c-s)} \delta_{N+1}
\end{aligned}
$$


The solution of matrix equation (15) with the dimensions $(N+1) \times$ $(N+1)$ is obtained by the way of Thomas algorithm. Once $\delta^{0}$ is determined, we can start the iteration of the system to find the parameters $\delta^{n}$ at time $t^{n}=n \Delta t$. Approximate solutions at the knots is found from the Eq. (5) and solution over the intervals $\left[x_{m}, x_{m+1}\right]$ is determined from the Eq.(15).

\section{Test Problems}

We have carried out three test problems to demonstrate the given algorithm. Accuracy of the method is measured by the error norm:

$$
L_{\infty}=\left\|u^{\text {exact }}-u^{\text {numeric }}\right\|_{\infty}=\max _{0 \leq j \leq N}\left|u_{j}^{\text {exact }}-u_{j}^{\text {numeric }}\right| .
$$

The RLW equation satisfy the following conservation laws which are corresponding to mass, momentum and energy [18]:

$C_{1}=\int_{-\infty}^{+\infty} u d x, C_{2}=\int_{-\infty}^{+\infty}\left(u^{2}+\mu\left(u_{x}\right)^{2}\right) d x, C_{3}=\int_{-\infty}^{+\infty}\left(u^{3}+3 u^{2}\right) d x$.

In numerical calculations, the conservation laws are calculated by use of the trapezoidal rule and the determination of $p$ in the exponential B-spline is made by experimentally.

\subsection{Propagation of single solitary wave}

The exact solution of RLW equation is given in [19] as follows:

$$
u(x, t)=\frac{3 c}{\cosh ^{2}\left(k\left(x-x_{0}-(1+\varepsilon c) t\right)\right)}
$$

where $k=\frac{1}{2} \sqrt{\frac{\varepsilon c}{\mu(1+\varepsilon c)}}$. This form of the solution is known as a single solitary wave with the amplitude $3 c$ and the velocity $1+\varepsilon c$. The initial condition is obtained by taking $t=0$ in Eq.116). We have used boundary conditions $\beta_{1}=0, \beta_{2}=0$. The values of the parameters seen in the above equations as

$$
c=0.1 \text { and } 0.03, x_{0}=0, \varepsilon=1, \mu=1 \text {. }
$$

With these parameters and the mentioned initial condition, the solitary wave moves across the interval $-40 \leq x \leq 60$ in time period $0 \leq t \leq$ 20. Similar with some early studies, space step $h=0.125$ and time step $\Delta t=0.1$ are used in numerical calculations. In this test problem, the $p=0.01262$ is determined by scanning the interval $[0,80]$ with the 

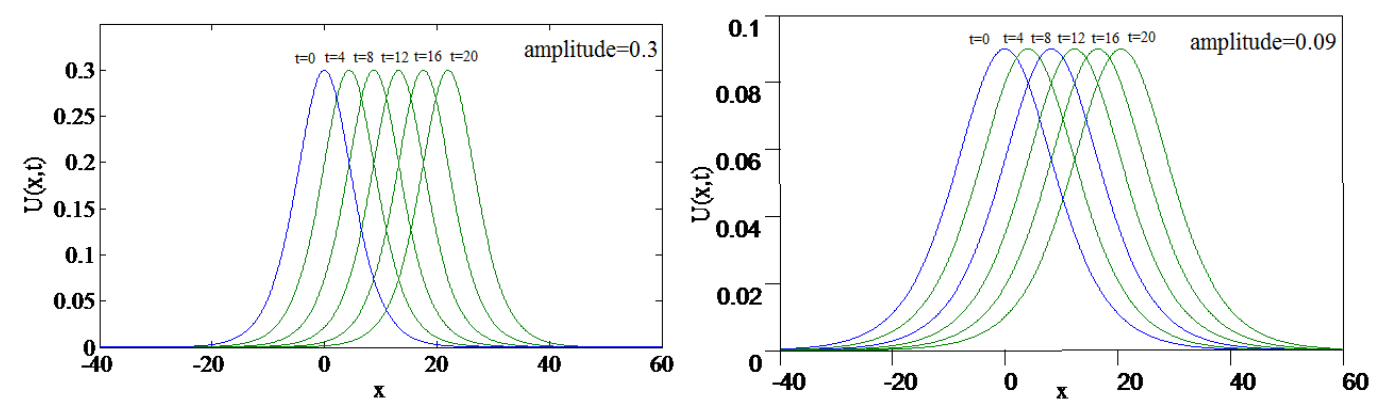

Figure 1: Solitary wave solutions

increment 0.1 first, then according to the results scanning the interval $[0,1]$ with the increment 0.00001 . The solution profiles are illustrated in Figure 1 at selected times. It is clear from this figure that the peak of the solitary wave remain kept during the running time.

The distribution of absolute error at $t=20$ for amplitude 0.3 and 0.09 is given in Figure 2, respectively. The maximum error for EBSGM occurs at the right hand boundary seen in Figure 2. We believe that this error arises due to magnitude of the wave and the physical boundary conditions to fit $u(a, 0) \approx 0$ and $u(b, 20) \approx 0$. If we extend the solution interval from $[-40,60]$ to $[-80,120], L_{\infty}$ error norm is seen to reduce from $0.4315 \times 10^{-3}$ to $0.63035 \times 10^{-5}$ at time $t=20$.
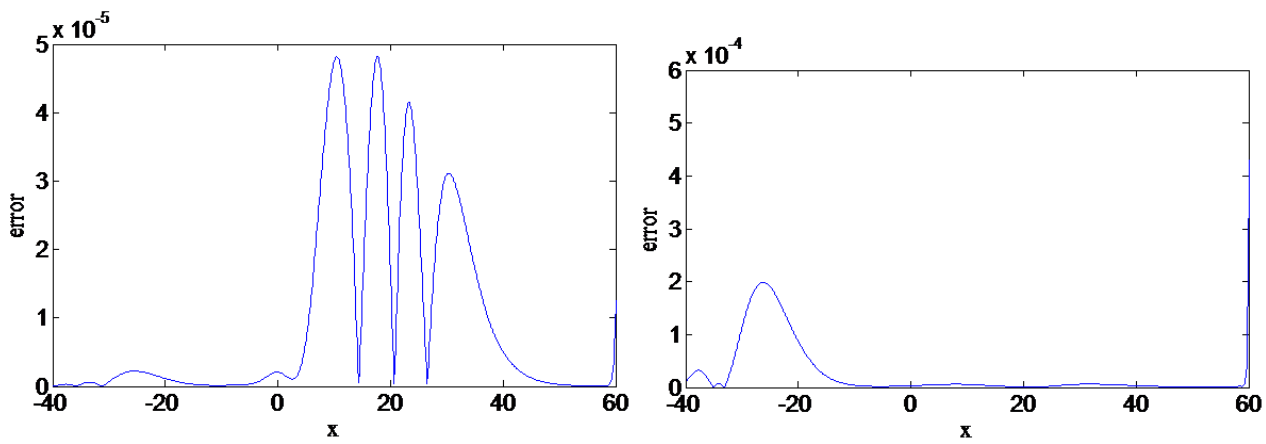

Figure 2: amplitude $=0.3$ and amplitude $=0.09$

The absolute error norms and the values of the conservation invariants $C_{1}, C_{2}, C_{3}$ are recorded in Table 2 and 3 for different amplitudes. To make a comparison with some early studies, the maximum errors with the conservation invariants are presented in Table 4 and 5. According to this tables, EBSGM is more accurate method than the some others. The values of the conservation invariants $C_{1}, C_{2}, C_{3}$ at different times remain fairly the same when compared with the analytical invariants $C_{1}=3.9799297, C_{2}=0.81046249, C_{3}=2.579007$ for amplitude 
0.3. When we take the amplitude as 0.09 , the value of the conservation invariant $C_{1}$ has some minor difference than the analytical value of it, whereas $C_{2}$ and $C_{3}$ are fairly same at different times.

Table 2: Errors and Invariants for amplitude $3 c=0.3$,

\begin{tabular}{ccccc}
\multicolumn{5}{c}{$h=0.125, \Delta t=0.1, p=0.01262$} \\
\hline \hline Time & $L_{\infty} \times 10^{5}$ & $C_{1}$ & $C_{2}$ & $C_{3}$ \\
\hline 0 & 0.0000 & 3.9799267 & 0.8104625 & 2.5790074 \\
4 & 1.268 & 3.9799299 & 0.8104625 & 2.5790075 \\
8 & 2.445 & 3.9799281 & 0.8104625 & 2.5790076 \\
12 & 3.418 & 3.9799255 & 0.8104626 & 2.5790077 \\
16 & 4.197 & 3.9799168 & 0.8104626 & 2.5790078 \\
20 & 4.820 & 3.9798827 & 0.8104626 & 2.5790079 \\
\hline
\end{tabular}

Table 3: Errors and Invariants for amplitude $3 c=0.09$,

\begin{tabular}{ccccc}
\multicolumn{5}{c}{$h=0.125, \Delta t=0.1, p=0.01262$} \\
\hline \hline Time & $L_{\infty} \times 10^{4}$ & $C_{1}$ & $C_{2}$ & $C_{3}$ \\
\hline 0 & 0.000 & 2.1070467 & 0.1273013 & 0.3888047 \\
4 & 2.302 & 2.1070975 & 0.1273011 & 0.3888041 \\
8 & 2.214 & 2.1068946 & 0.1273011 & 0.3888041 \\
12 & 2.129 & 2.1065454 & 0.1273011 & 0.3888040 \\
16 & 2.139 & 2.1053719 & 0.1273012 & 0.3888032 \\
20 & 4.315 & 2.1045885 & 0.1273012 & 0.3888023 \\
\hline
\end{tabular}

Table 4:

Errors and Invariants for amplitude $3 c=0.3, h=0.125, \Delta t=0.1, p=0.01262$

\begin{tabular}{ccllll}
\hline \hline Method & Time & $L_{\infty} \times 10^{3}$ & \multicolumn{1}{c}{$C_{1}$} & \multicolumn{1}{c}{$C_{2}$} & \multicolumn{1}{c}{$C_{3}$} \\
\hline Analytical & & & 3.97995 & 0.8104624 & 2.579007 \\
\hline EBSGM & 20 & 0.04820 & 3.979883 & 0.8104626 & 2.579008 \\
{$[14$} & 20 & 0.02643 & 3.979909 & 0.8104625 & 2.579007 \\
{$[16$} & 20 & 0.07337 & 3.979883 & 0.8104612 & 2.579003 \\
{$[12$} & 20 & 1.56640 & 3.961597 & 0.804185 & 2.558292 \\
{$[13$} & 20 & 0.10299 & 3.979858 & 0.8104596 & 2.578999 \\
{$[2]$} & 20 & 0.116 & 3.979883 & 0.8102762 & 2.578393 \\
{$[23$} & 20 & 0.91465 & 3.97972 & 0.81026 & 2.57873 \\
{$[2]$} & 20 & 0.07344 & 3.979888 & 0.8104622 & 2.579006 \\
{$[25$} & 20 & 0.073 & 3.97989 & 0.81046 & 2.57901 \\
\hline
\end{tabular}


Table 5:

Errors and Invariants for amplitude $3 c=0.09, h=0.125, \Delta t=0.1, p=0.01262$

\begin{tabular}{llllll}
\hline \hline Method & Time & $L_{\infty} \times 10^{3}$ & \multicolumn{1}{c}{$C_{1}$} & \multicolumn{1}{c}{$C_{3}$} & \multicolumn{1}{c}{$C_{3}$} \\
\hline Analytical & & & 2.109407 & 0.12730171 & 0.3888059 \\
\hline EBSGM & 20 & 0.431512 & 2.104589 & 0.12730121 & 0.3888023 \\
{$[12$} & 20 & 1.5506 & 2.128869 & 0.127228 & 0.388571 \\
{$[22$} & 20 & 0.432 & 2.104584 & 0.12729366 & 0.3887776 \\
{$[23$} & 20 & 0.439145 & 2.10902 & 0.12730 & 0.38880 \\
{$[24$} & 20 & 0.19806 & 2.104708 & 0.1273006 & 0.3888025 \\
{$[25$} & 20 & 0.199 & 2.10467 & 0.12730 & 0.38880 \\
\hline
\end{tabular}

\subsection{Interaction of two solitary waves}

In this section, we will study the interaction of two solitary waves having different amplitudes and moving in the same direction. The initial condition is

$$
\begin{aligned}
u(x, 0) & =u_{1}+u_{2}, \\
u_{j} & =\frac{3 A_{j}}{\cosh ^{2}\left(k_{j}\left(x-\widetilde{x}_{j}\right)\right)}, \quad A_{j}=\frac{4 k_{j}^{2}}{1-4 k_{j}^{2}}, \quad j=1,2
\end{aligned}
$$

where the following parameters are chosen to coincide values in the literature:

$$
\varepsilon=1, \mu=1, k_{1}=0.4, k_{2}=0.3, \widetilde{x}_{1}=15, \widetilde{x}_{2}=35 \beta_{1}=0, \beta_{2}=0 .
$$

Initially, these parameters yields the solitary waves with the amplitudes 5.333375 and 1.687502 positioning around points $x=15$ and $x=35$ respectively. The computation is carried out up to time $t=30$ with time step $\Delta t=0.1, N=400$ over the finite interval $[0,120]$ and $p$ is selected as 1 for EBSGM.

Numerical solutions of $u(x, t)$ at various times are depicted in Figure 3, and the initial solution has been propagated rightward. It is seen from the Figure 3 that the solitary waves are subjected to a collision about time $t=15$ and after the interaction they propagate with their original amplitudes to the right seeing at time $t=30$.

The conservation invariants are presented at some selected times in Table 6. According to this table, during the interaction there are some changes at the conservation constants $C_{2}$ and $C_{3}$ whereas the constant $C_{1}$ remain nearly same. 


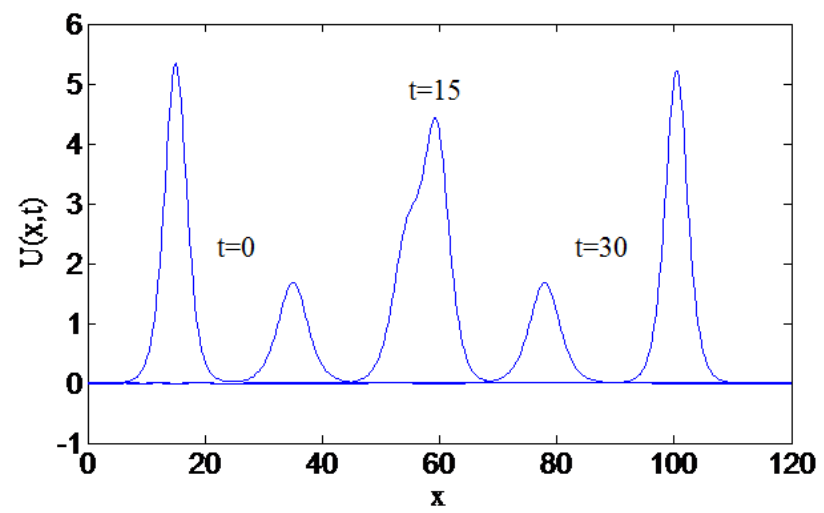

Figure 3: Interaction of two positive solitary waves at $t=0,15$ and 30.

Table 6: Invariants for $h=0.3, \Delta t=0.1, p=1$.

\begin{tabular}{cccc}
\hline \hline Time & $C_{1}$ & $C_{2}$ & $C_{3}$ \\
\hline 0 & 37.916502 & 120.522247 & 744.081209 \\
5 & 37.861378 & 119.772286 & 737.819263 \\
10 & 37.810403 & 119.129874 & 732.319072 \\
15 & 37.790851 & 119.012803 & 730.931470 \\
20 & 37.752779 & 118.312087 & 725.698903 \\
25 & 37.701304 & 117.600957 & 719.868731 \\
30 & 37.650120 & 116.909863 & 714.174760 \\
\hline \hline
\end{tabular}

\subsection{Wave generation}

An applied force like an introduction of fluid mass, an action of some mechanical device, to a free surface, will induce waves. In this numerical experiment, we take following boundary condition to generate waves with the RLW equation.

$$
u(a, t)=\beta_{1}= \begin{cases}U_{0} \frac{t}{\tau}, & 0 \leq t \leq \tau \\ U_{0}, & \tau<t<t_{0}-\tau \\ U_{0} \frac{t_{0}-t}{\tau}, & t_{0}-\tau \leq t \leq t_{0} \\ 0, & \text { otherwise }\end{cases}
$$

and $u(b, t)=\beta_{2}=0$ is studied to generate waves. This forced boundary condition known as a wave maker at one end.

The parameters $U_{0}=2, \Delta t=0.1, h=0.4, t_{0}=20, \tau=0.3$ are chosen to make a comparison with earlier works over the region $0 \leq x \leq 260$. $p$ is selected as 1 for EBSGM. During the run time of the algorithm, five solitary waves are produced. Although first four waves 
have reached amplitudes larger than forcing amplitudes, the last one is less than that of the forcing one. When forcing is switched off, the last wave has not enough time to evolve. Subsequently, no new wave are born. A view of travelling solitary wave is presented at time $t=100$ in Figure 4. Amplitudes of solitary waves versus time are depicted in Figure 5. At various time, amplitudes of the solitary waves and the conservation constants are demonstrated in Table 7 for EBSGM. In addition to this, other amplitudes of the solitary waves which are reduced the other studies are shown in Table 7 for time $t=100$. Our results are in conformity with that of studies [10, 34, 25].

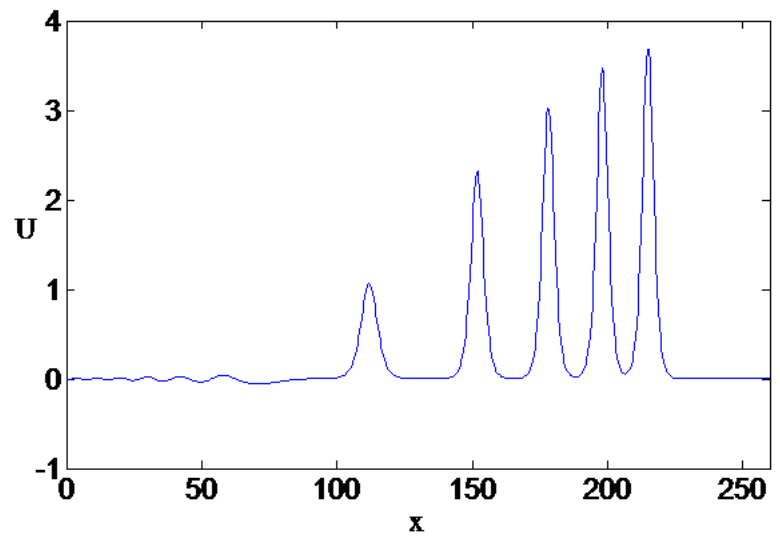

Figure 4: Solitary wave produced by boundary forcing of duration $t_{0}=20$ and amplitude $U_{0}=2$ at time $t=100, h=0.4, \Delta t=0.1, p=1$.

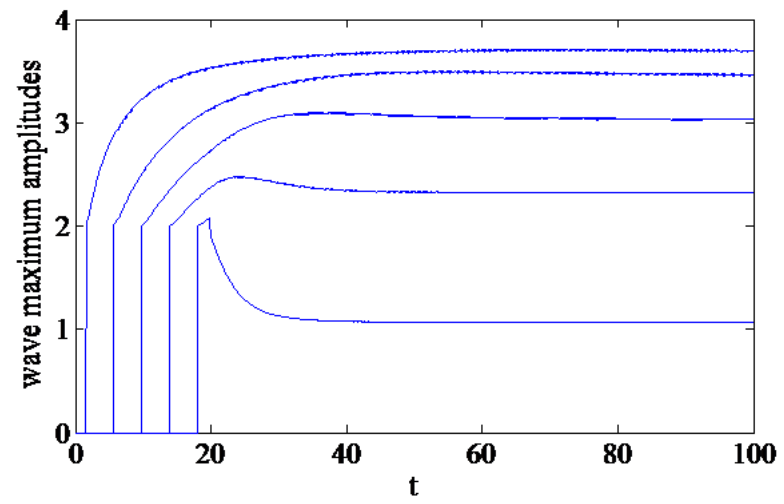

Figure 5: Evolution of wave amplitudes. 
Table 7: Solitary wave amplitude with $U_{0}=2, h=0.4, \Delta t=0.1, p=1$ and period of forcing $0 \leq t_{0} \leq 20,0 \leq x \leq 260$.

\begin{tabular}{lcccccrrr}
\hline \hline \multicolumn{1}{c}{ Time } & 1 & 2 & 3 & 4 & 5 & \multicolumn{1}{c}{$C_{1}$} & \multicolumn{1}{c}{$C_{2}$} & \multicolumn{1}{c}{$C_{3}$} \\
\hline 2.5 & 2.26 & & & & & 8.9397 & 16.1942 & 74.8986 \\
5 & 2.78 & & & & & 19.3958 & 41.3363 & 206.5459 \\
7.5 & 3.05 & 2.24 & & & & 29.3109 & 64.3064 & 324.6697 \\
10 & 3.23 & 2.53 & 2.02 & & & 39.2124 & 87.2152 & 442.2752 \\
15 & 3.44 & 2.91 & 2.43 & 2.06 & & 59.2209 & 133.8372 & 682.1665 \\
20 & 3.53 & 3.14 & 2.73 & 2.38 & 1.90 & 76.8502 & 175.1059 & 882.7008 \\
40 & 3.67 & 3.46 & 3.08 & 2.35 & 1.08 & 78.5060 & 174.3022 & 882.6289 \\
60 & 3.70 & 3.50 & 3.05 & 2.33 & 1.07 & 78.4275 & 173.5305 & 877.6376 \\
80 & 3.70 & 3.48 & 3.03 & 2.33 & 1.07 & 78.3394 & 172.7643 & 872.6609 \\
100 & 3.68 & 3.47 & 3.03 & 2.32 & 1.07 & 78.2589 & 172.0094 & 867.7610 \\
$100[\overline{10}]$ & 3.76 & 3.52 & 3.06 & 2.33 & 1.07 & & & \\
$100[\overline{25}]$ & 3.77 & 3.52 & 3.08 & 2.38 & 1.18 & & & \\
$100[34]$ & 3.76 & 3.51 & 3.07 & 2.31 & 0.98 & & & \\
\hline
\end{tabular}

\section{Conclusion}

In this paper, we investigate the utility of the exponential B-spline algorithm for solving the RLW equation. The efficiency of the method is tested on the propagation of the single solitary wave, the interaction of two solitary waves and wave generation. To see the accuracy of the method, $L_{\infty}$ error norm and conservation quantities $C_{1}, C_{2}$ and $C_{3}$ are documented based on the obtained results. Exponential B-spline based method gives accurate and reliable results for solving the RLW equation. For the first test problem, EBSGM leads to more accurate results than the collocation-based method but similar results with the some Galerkin methods. In the second test problem, there is no exact solution therefore simulation is shown graphically and the conservation quantities are tabulated. Generation of waves by using variable boundary conditions at left has been achieved and wave profiles and their amplitudes are documented. In conclude, the numerical algorithm in which the exponential B-spline functions are used, performs well compared with other existing numerical methods for the solution of RLW equation.

\section{Acknowledgements}

The author, Melis Zorşahin Görgülü, is grateful to The Scientific and Technological Research Council of Turkey for granting scholarship for PhD studies and all of the authors are grateful to The Scientific and Technological Research Council of Turkey for financial support for their project. 


\section{References}

[1] T. B.Benjamin, J. L.Bona and J. J.Mahony, Model equations for long waves in non-linear dispersive systems. Phil. Trans. Roy. Soc., London, A272 (1972), 47-78.

[2] M. E.Alexander and J. L.Morris, Galerkin methods applied to some model equations for non-linear dispersive waves. J. Comput. Physics, 30 (1979), 428-451.

[3] P. J. Jain and L. Iskandar, Numerical solutions of the regularized long wave equation, Comput. Meth. Appl. Mech. Eng., 20 (1979), 195-201.

[4] B. J. McCartin, Theory, computation and application of exponential splines, Courant Mathematics and Computing Laboratory Research and Development Report, DOE/ER/03077-171,(1981).

[5] G. Ben-Yu and V.S.Manoranjan, A spectral method for solving the RLW equation, IMA J. Numer. Anal., 5 (1985), 307-318.

[6] B.Guo and W.Cao, The fourier pseudospectral method with a restrain operator for the RLW equation. J. Comput. Physics, 74 (1988), 110-126.

[7] M. Sakai and R. A. Usmani, , A class of simple exponential B-splines and their application to numerical solution to singular perturbation problems, Numer. Math. vol. 55, (1989),pp. 493-500.

[8] P. C.Jain, R.Shankar and T. V. Singh, Numerical solution of Regularized long-wave equation, Commum. Numer. Methods Eng, 9 (1993), 579-586.

[9] L. R. T. Gardner, G. A. Gardner and I.Dag, A B-spline finite element method for the regularized long wave equation, Commun. Numer. Meth. Eng., 11 (1995), 59-68.

[10] L. R. T. Gardner and I.Dag, The boundary-forced regularised long wave equation, Il Nuovo Cimento B, 110:12 (1995), 1487-1496.

[11] D.Bhardwaj and R.Shankar, A computational method for Regularised Long Wave Equation. Comp.Math. Appl., 40 (2000), 13971404.

[12] I.Dag and M.N.Ozer, Approximation of the RLW equation by the least square cubic B-spline finite element method, Applied Mathematical Modelling, 25 (2001), 221-231.

[13] I.Dag, A.Dogan and B.Saka, B-spline collocation methods for numerical solutions of the RLW equation, Intern. J. Computer Math., 80 (2003), 743-757.

[14] P.Avilez-Valente, F.J.Seabra-Santos, A Petrov-Galerkin finite element scheme for the regularized long wave equation, Computational Mechanics, 34 (2004), 256-270.

[15] S.Kutluay and A.Esen, A finite difference solution of the regularized 
long-wave equation, Mathematical Problems in Engineering, (2006), $1-14$.

[16] I.Dag, B.Saka and D.Irk, Galerkin method for the numerical solution of the RLW equation using quintic B-splines, Journal of Computational and Applied Mathematics, 190 (2006), 532-547.

[17] Z.Luo and R.Liu, Mixed finite element analysis and numerical solitary solution for the RLW equation. SIAM. J. Numer. Anal., 36(1) (1998), 89-104.

[18] P. J.Olver, Euler operators and conservation laws of the BBM equation. Math. Proc. Camb. Phil. Soc., 85 (1979), 143-159.

[19] D. H.Peregrine, Calculations of the development of an undular bore, J. Fluid. Mech., 25(2) (1966), 321-330.

[20] J. M.Sanz Serna and I. C.Petrov, Galerkin methods for non linear dispersive wave. J. Comput. Physics, 39 (1981), 94-102.

[21] D. M. Sloan, Fourier pseudospectral solution of the regularised long wave equation, J. Comput. Appl. Math., 36 (1991), 159-170.

[22] I. Dag, B. Saka and D. Irk, Application of cubic B-splines for numerical solution of the RLW equation, Applied Mathematics and Computation, 159 (2004), 373-389.

[23] L. Mei, Y. Chen, Numericel solution of RLW equation using Galerkin method with extrapolation techniques, Computer Physics Communications, 183 (2012), 1609-1616.

[24] B. Saka, I. Dag, A numerical solution of the RLW equation by Galerkin method using quartic B-splines, Communications in numerical methods in engineering, 24 (2008), 1339-1361.

[25] B. Saka, I. Dag and A. Dogan, Galerkin method for the numerical solution of the Rlw equation using quadratic B-splines, International Journal of Computer Mathematics, 81:6 (2004), 727-739.

[26] B. J. McCartin, Theory of exponential splines, Journal of Approximation Theory,, 66,1-23 (1991), 86-96.

[27] D. Amos, Computation with splines and B-splines, SAND, 78-1968 (1979).

[28] C. De Boor, A practical guide to splines, Springer-Verlag, (1978).

[29] P. M. Pruess, Splines and variational methods, Wiley, (1975).

[30] S. Pruess, An algorithm for computing smoothing splines in tension, Computing, 19(1978), 365-373.

[31] S. C. S. Rao, M. Kumar, Exponential B-spline collacation method for self-adjoint singularly perturbed value problems, Applied Numerical Mathematics, 59(2008), 1572-1581.

[32] D. Radunovic, Multiresolution exponential B-splines and singularly perturbed boundary problem, Numer Algor 47, 191-210, 2008.

[33] R. Mohammadi, Exponential B-spline solution of convection- 
diffusion equation, Applied Mathematics, 4(2013), 933-944.

[34] Q. Chang, G. Wang and B. Guo, Conservative scheme for a model of nonlinear dispersive waves and its solutary waves induced by boundary motion, J. Comput. Phys., 93(1991), 360-375. 